\title{
Comparison between Total Echo Score and Total Commissural Morphology Score for the Outcome and Complication of Balloon and Surgical Closed Mitral Commissurotomy
}

\author{
AK Choudhury, S Alam, GM Faruque, M Ali, NAM Momenuzzaman, S Rahman
}

Department of Cardiology, National Institute of Cardiovascular Diseases, Dhaka

Keywords:
PTMC,
CMC,
Mitral stenosis,
Rheumatic
heart disease

\begin{abstract}
Background: Now a days mitral balloon valvoplasty(PTMC) is an alternative to closed surgical mitral commissurotomy (CMC) for the treatment of selectcd patients with rheumatic mitral stenosis. To compare between the total echo score (Wilkin's score) total echocardiographic commissural morphology score (TC) for outcome and as a predictors of complications of both procedures.
\end{abstract}

Method: We carried out a prospective well matched comparative observational study on 123 patients of symptomatic mitral and three patients were rejected due to procedural complications and technical failure.

Result: Age ranges were 1255 years, mean $( \pm S D)$ age was 28.83+9.33 years. Out of 120 patients, 41 $(34.2 \%)$ were male and $79(65.8 \%)$ were female. Before procedure, $29(48.3 \%)$ and $32(53.3 \%)$ patients were in NYHA class III.Total Wilkins score was in the range of 4 10. Mean $( \pm S D)$ of total Wilkins score were 6.43+1.53 and 6.30+1.33. Good commissural morphology (score 0 1) were present in 38 $(63.5 \%)$ and $36(60 \%)$ and bad commissural morphology (score 23$)$ were present in 22 (26.7 \%) and. $24(40 \%)$ in both groups respectively.

Mitral valve area increased from a mean $( \pm S D)$ of $0.80 \pm 0.16$ and $0.79 \pm 0.15$ to $1.94 \pm 0.24$ and $1.92 \pm$ $0.26 \mathrm{~cm}^{2}$ in PTMC and CMC groups respectively. Transmitral mean and peak pressure gradient also decreased significantly in both the individual procedures but no statistically significant difference between the procedures.

NYHA class improved by class 1 or more in most patients in both groups. There were 2 (3.33\%) cases of cardiac temponade due cardiac perforation in PTMC group, of which one need repair and CMC and another was managed conservatively. There were also $3(5 \%)$ patients in PTMC and I (1. $66 \%)$ patient in CMC developed peripheral thromboembolism and one patient (1.66\%) developed arteriovenous fistula in PTMC group. Mitral regurgitation grade III, developed in 3 (5\%) patients and one patient (1.66\%) in PTMC and CMC respectively having no statistical significance.

Conclusion: Total Wilkin's score and total commissural morphology score were found to be most important preprocedural variable associcated with the outcome and as a predictors of post procedural complications

(CVJ 2008; 1(1) : 34-43)

\section{Introduction}

To compare the immediate and early outcome of percutaneous transvenous mitral commissurotomy (PTMC) and surgical closed mitral commissurotomy (CMC) in relation to valve morphology in Bangladesh and to establish whether only commissural morphology or total valve score would be the determinant factor in the outcome of both procedures. In this study we also compare the sensitivity of different echocardiographic scoring systems to assess the outcome and to avoid the complications of PTMC and CMC.

Dr. Kanji Inoue was the first to perform percutaneous transvenous mitral commissurotomy (PTMC) as an alternative to surgery in the treatment of mitral stenosis.

Address Correspondence : Amal Kumar Choudhury, Assistant Professor, Department of Cardiology, NICVD, Dhaka, amaldr@yahoo.com 
Elliot Cutler performed the first closed mitral commissurotomy (CMC) with a tenotomy knife on a 12 years old patient with rheumatic mitral stenosis in 1923. Despite initial enthusiasm, mortality rates were excessive and the procedure was abandoned in 1929. It was not until the late 1940s, that Harken and Bailey described the closed technique of mitral commissurotomy, which became the accepted surgical treatment for mitral stenosis. The primary mechanism of both is similar and involves a mechanical splitting of one or both mitral commissures.

For about last 16 years, the only treatment that was available for these patients was surgical commissurotomy, either open or closed.

Clearly, there will be difficulty in comparing the techniques since there are variabilities in the patient populations that undergo these procedures due to valve pathology and the use of patients from various countries; where the patient's ages and valve pathology are not the same as that seen with RHD in other countries.

We believe that despite the early success of mitral balloon valvuloplasty, it should remain in investigational technique at the current time. Surgical commissurotomy is a highly successful technique with a long record of experience, whereas the long term outcome of balloon valvuloplasty is not yet known and the rate of certain complications may be higher. In addition, the criteria for optimal patient selection for balloon mitral valvuloplasty is not yet known and the rate of certain complications may be higher. In addition, the criteria for optimal patient selection for balloon mitral valvuloplasty are still evolving and technical aspects of the procedure are changing. ${ }^{1}$

\section{Materials and Methods \\ Study population}

This prospective comparative study was carried on 60 patients in each group of symptomatic mitral stenosis, who attended the Department of Cardiology and Cardiovascular Surgery, during the period from January 1998 to July 1999 in the Department of Cardiology and Cardiovascular Surgery, National Institute of Cardiovascular Diseases (NICVD), Dhaka, Bangladesh.

\section{Preprocedural evaluation}

Echocardiography:

- Echo was done by one expert using $2.8 / 3.5 \mathrm{MHz}$ convex transducer.

- Mitral valve area was calculated by planimetry method in short-axis view and also by pressure half- time(PHT) method.

- Mitral valve morphology was studied extensively by several methods.

- Mitral valve echo-score (Wilkins'score)were developed by Abascal et al.(1988) (Table-1) and a score more than 10 were excluded.

- Only commissural morphology of mitral valve was studied considering fusion, thickness and calcification of the commissures by Fatkin et $a l .^{2}$

- MR echo score by Padial et al. was developed to predict the development of mitral regurgitation as a complication of the procedures ${ }^{3}$.

- Mean and peak pressure gradients across the mitral valve were calculated using CW Doppler.

- Pulmonary artery systolic pressure (PASP) was measured considering the tricuspid regurgitation by CW Doppler.

- Colour flow imaging was done. Patients with > grade II MR, > grade II AR and significant aortic stenosis were excluded.

- 2D, M mode study: LA diameter was calculated and LV study was done, including LVEF\% by POMBO method from M mode echocardiography.

- TEE was done before procedures in suspected cases of LA thrombus.

Procedure(PTMC and CMC)

1) PTMC was done with all aseptic precautions through femoral approach under local anaesthesia.

2) Following pressure studies were done during the procedure: aorta, LV, RV, PA and LA; LV/ LA gradient calculated.

3) A simple balloon sizing method based on body height for selection of appropriate size balloon catheter was used:

Balloon size $=\frac{\text { Height in cm }}{10}+10$ 
4) $\mathrm{CMC}$ was done as closed heart surgery under general anaesthesia by skin incision through left 4 th of 5 th intercostal space. Transatrial finger fracture technique and transventricular commissurotomy technique using Tubbs' dilator were applied. ${ }^{4}$

5) The width of Tubbs' dilator was set in advance in 3.0 to $3.5 \mathrm{~cm}$ for male and in 2.5 to $3.0 \mathrm{~cm}$ for female.

6) Any periprocedural complication was recorded.

\section{Postprocerure evaluation}

1) $2 \mathrm{D}, \mathrm{M}$ mode, $\mathrm{PW}, \mathrm{CW}$ Doppler and colour flow imaging were done iminediately after PTMC and within $7 \pm 3$ days after $\mathrm{CMC}$ to evaluate all echocardiographic parameters, which were taken before the procedures.

2) Successful procedure was defined as increased in mitral valve area (MVA) to $>1.5 \mathrm{~cm}^{2}$ or $>50$ percent increase in MVA.

\section{Follow-up evaluation}

1) Patients were followed up at 1,3 and 6 months after PTMC. During follow up, history taking, clinical examination were done. $2 \mathrm{D}, \mathrm{M}$ mode echocardiography and CW Doppler study and colour flow imaging were done at each follow up. Mitral valve area in planimetry and PHT method were recorded. Mean and peak pressure gradient across mitral valve, PASP, degree of MR, TR, LV study and ASD flow were studied.

2) Restenosis (echocardiographic) was defined as loss of ${ }^{3} 25$ percent of mitral valve area acquired after PTMC or CMC or MVA reduced to $<1.5$ $\mathrm{CM}^{2}$.

\section{Statiscal analysis}

This study was a prospective randomized comparative observational study, comparing the role of two echocardiographic scoring systems as predictors of outcome and complications of both the procedures in Bangladesh. Comparison of different echocardiographic parameters and other variables between PTMC and CMC, before and after procedures were made using paired or unpaired Student's 'T' test or Chi square $\left(\mathrm{X}^{2}\right)$ test.Both computer programme (SPSS) and manual technology were applied for statistical analysis. Probability $(\mathrm{P})$ value $<0.05$ was considered as significant.

\section{Observations and Results}

Echocardiography: Two dimensional, M mode, spectral and colour Doppler study were done in all the patients, both before and after PTMC and CMC.

Mitral valve area (MVA): Mitral valve area was measured by both planimetry and pressure half time method. MVA was in the range of $0.501 .20 \mathrm{~cm}$ ' with mean value of $0.79 \mathrm{~cm}^{2}$. Between the two methods, there was no statistical difference. Most of the patients, i.e. 114 (95\%) had MVA of $1 \mathrm{CM}^{2}$ or less, ,only 6 (5\%) patients had MVA more than I $\mathrm{cm}^{2}$. There was no statistically significant difference of the mean value of MVA between PTMC and CMC groups.

Mean pressure grading (MPG): MPG were measured using continuous wave Doppler method in apical four chamber view. MPG 'were in the range of 633 rnmHg with mean $( \pm \mathrm{SD}) 18.37+6.83 \mathrm{mmHg}$. Ninety percent patients had MPG of $10 \mathrm{mmHg}$ and above. There was no statistically significant difference in the preprocedural MPG between PTMC and CMC group.

Peak pressure gradient (PPG): PPG were measured using the same procedure as MPG. PPG were found in the range of $1348 \mathrm{mmHg}$, with mean $( \pm \mathrm{SD})$ 30.28+9.33 MMhg. One hundred sixteen patients (96.7\%) had PPG $17 \mathrm{mmHg}$ and above. Between PTMC and CMC group, there was no significant difference of mean PPG.

Pulmonary artery systolic pressure (PASP): PASP were obtained by tricuspid regurgitation (TR) gradient plus $10 \mathrm{mmHg}$. PASP were found in the range of $29116 \mathrm{mmHg}$, with mean $( \pm \mathrm{SD})$ 76.43+18.84 mmHg. Between PTMC and CMC group, there was no significant difference of PASP was observed before procedures.

Left atrial diameter (LAD): LADs were measured by $\mathrm{M}$ mode. Range was $3770 \mathrm{~mm}$, with a mean $( \pm$ SD) $48.30+6.97 \mathrm{~mm}$. Ninety five percent patients had LAD $40 \mathrm{~mm}$ and above, with no difference of LAD between the two groups. Presence of thrombus and spontaneous echo contrast in LA and LA appendages were ruled out using different views.

Mitral regurgitation (MR): By using spectral Doppler and colour flow imaging, presence and grade of MR were assessed in all patients. No MR was found in 39 (65\%) and 46 (76.7\%) patients of PTMC and CMC groups, respectively. Grade I MR were present in 19 
(31.7\%) and 14 (23.3\%) patients of PTMC and CMC group, respectively, before undergoing procedures.

Echocardiographic score of mitral valve morphology In our study, to assess the preprocedural status of mitral valve morphology, following three echo scoring systems had been follows:

Wilkins'echocardiographic score of mitral valve Mobility of leaflet: Mobility scores were in the range of 03 with no patient with a score of 4 . Most of the patients, 57 (45\%) patients of PTMC group and 58 (96.7\%) patients of CMC group, had score of 1 and 2. Mean $( \pm \mathrm{SD})$ score for mobility were $1.82+0.47$ and $1.80+0.51$ in PTMC and CMC group, respectively, with no significant difference.

Thickness of mitral leaflets: All the patients had score within the range of 13 with no patient with score 0 and 4 . Most of the patients (95\%) of both groups had score of 1 and 2 with no significant difference.

Subvalvular changes (SVC): Most of the patients (75\%) had SVC of score 2 and 3. One patient of PTMC group had SVC score of 4.

Calcification: More than 55 percent patients of both groups had no calcification (score 0 ). Two patients of PTMC group had calcification score of 3 . None had a score of 4 .
Total Wilkins'score: Total score was calculated by adding the subscores of above mentioned variables of mitral valve apparatus. Total score was in the range of 410 . Mean $( \pm \mathrm{SD})$ of total score were $6.43+1.53$ and 6.30+1.33 in PTMC and CMC group, respectively. Twenty six $(21.6 \%)$ patients had a total score of 810 . Most of the patients (55\%) of both groups had a total score of 67 .

Commissural morphology score ${ }^{2}$

Only commissural morphology of mitral valve was assessed by commissural fusion, thickness. (fibrosis) and calcification, each of three component having score 0 1. So minimum total commissural morphology score was 0 in 8 (6.7\%). Good (score 01 ) commissural morphology was $38(63.3 \%)$ and 36 $(60 \%)$ in PTMC and CMC group, respectively. Bad (score 2 3) commissural morphology was 22 (36.7 \%) and 24 (40\%) in PTMC and CMC group, respectively.

Outcome of procedures in the context of Echocardiographic mitral valve morphology

In our study, one important target was to observe the association between the preprocedural mitral valve morphology by different echo scoring system and postprocedural outcome of the two procedures, and also compare the different echo scoring system of mitral valve morphology with the postprocedural outcome.

Table-I

Comparison of changes between mitral valve area (MVA) and total Wilkins'echo score before and after procedure.

\begin{tabular}{|c|c|c|c|c|c|c|}
\hline \multirow{2}{*}{$\begin{array}{l}\text { Wilkins' } \\
\text { Total } \\
\text { echo } \\
\text { score }\end{array}$} & \multicolumn{3}{|c|}{ Pre MVA $\left(\mathrm{cm}^{2}\right)$} & \multicolumn{3}{|c|}{ Post MVA $\left(\mathrm{cm}^{2}\right)$} \\
\hline & $\begin{array}{c}\text { PTMC } \\
(\text { Mean } \pm \mathrm{SD})\end{array}$ & $\begin{array}{c}\mathrm{CMC} \\
(\mathrm{Mean} \pm \mathrm{SD})\end{array}$ & P value & $\begin{array}{c}\text { PTMC } \\
(\text { Mean } \pm \text { S D) }\end{array}$ & $\begin{array}{c}\mathrm{CMC} \\
\text { (Mean+SD) }\end{array}$ & Pvalue \\
\hline$<6$ & $0.82 \pm 0.19$ & $0.80 \pm 0.15$ & 0.532 & $2.02+0.33$ & $1.93+0.22$ & $0.163^{\mathrm{NS}}$ \\
\hline${ }^{3} 6$ & $0.79 \pm 0.14$ & $0.78 \pm 0.15$ & 0.776 & $1.91+0.19$ & $1.85 \pm 0.36$ & $0.590^{\mathrm{NS}}$ \\
\hline
\end{tabular}

"NSNot significant

Table-II

Distribution of echocardiographic commissural morphology of mitral valve of the patients.

\begin{tabular}{|c|c|c|c|c|c|}
\hline \multirow[b]{2}{*}{ Class } & \multicolumn{2}{|c|}{ PTMC $(\mathrm{n}=60)$} & \multicolumn{2}{|c|}{$\mathrm{CMC}(\mathrm{n}=60)$} & \multirow[b]{2}{*}{ P value } \\
\hline & Frequency & $(\%)$ & $\overline{\text { Frequency }}$ & $\overline{(\%)}$ & \\
\hline $\begin{array}{l}\text { Good } \\
\text { (score 0-1) }\end{array}$ & 38 & $(63.3)$ & 36 & $(60.0)$ & $0.113^{\mathrm{NS}}$ \\
\hline $\begin{array}{l}\text { Bad } \\
\text { (score 2-3) }\end{array}$ & 22 & $(36.7)$ & 24 & $(40.0)$ & $0.183^{\mathrm{NS}}$ \\
\hline
\end{tabular}

NSNot significant 


\section{Table-III}

Comparison of change between mitral valve area (MVA) and total echocardiographic commissural morphology (TC morph)'before and after procedure

\begin{tabular}{|c|c|c|c|c|c|c|}
\hline \multirow{2}{*}{$\begin{array}{l}\text { TC } \\
\text { morph } \\
\left(\begin{array}{l}03)\end{array}\right.\end{array}$} & \multicolumn{3}{|c|}{ Pre MVA $\left(\mathrm{cm}^{2}\right)$} & \multicolumn{3}{|c|}{ Post MVA $\left(\mathrm{cm}^{2}\right)$} \\
\hline & $\begin{array}{c}\text { PTMC } \\
(\text { Mean } \pm \text { SD) }\end{array}$ & $\begin{array}{c}\mathrm{CMC} \\
(\mathrm{Mean} \pm \mathrm{SD})\end{array}$ & Pvalue & $\begin{array}{c}\text { PTMC } \\
(\text { Mean + SD) }\end{array}$ & $\begin{array}{c}\text { CMC } \\
(\text { Mean+ SD) }\end{array}$ & P value \\
\hline $\begin{array}{l}\text { Good } \\
\left(\begin{array}{ll}0 & 1\end{array}\right)\end{array}$ & $0.90 \pm 0.18$ & $0.85+0.16$ & $0.866 \mathrm{NS}$ & $1.99+0.31$ & $220+0.24$ & $0.28 \mathrm{I}^{\mathrm{NS}}$ \\
\hline $\begin{array}{l}\mathrm{Bad} \\
(23)\end{array}$ & $0.70+0.14$ & $0.79 \pm 0.13$ & $0.949 \mathrm{NS}$ & $1.90+0.19$ & $1.94+0.24$ & $0.647^{\mathrm{NS}}$ \\
\hline
\end{tabular}

Table-IV

Distribution of Wilkins' echocardiographic scores of mitral valve morphology among the patients

\begin{tabular}{|c|c|c|c|c|}
\hline \multirow[b]{2}{*}{ Parameters } & \multicolumn{2}{|c|}{ PTMC $(n=60)$} & \multicolumn{2}{|c|}{ CMC $(n=60)$} \\
\hline & Frequency & $(\%)$ & Frequency & $(\%)$ \\
\hline \multicolumn{5}{|l|}{ Mobility } \\
\hline 0 & 1 & $(1.7)$ & 1 & $(1.7)$ \\
\hline 1 & 12 & $(20.0)$ & 10 & $(16.7)$ \\
\hline 2 & 45 & $(75.0)$ & 48 & $80.0)$ \\
\hline 3 & 2 & (3.3) & 1 & $(1.7)$ \\
\hline Mean_SD & $1.82 \pm 0.47$ & $1.80 \pm 0.51$ & & \\
\hline \multicolumn{5}{|c|}{$\mathrm{P}$ value $=0.853$ (not significant) } \\
\hline \multicolumn{5}{|c|}{ Thickness } \\
\hline 1 & 9 & $(15.0)$ & 7 & $(11.7)$ \\
\hline 2 & 48 & $(80.0)$ & 52 & $(86.7)$ \\
\hline 3 & 3 & $(50.0)$ & 1 & $(1.7)$ \\
\hline Mean_SD & \multicolumn{2}{|c|}{$1.90 \pm 0.44$} & \multicolumn{2}{|c|}{$1.90 \pm 0.35$} \\
\hline \multicolumn{5}{|c|}{$\mathrm{P}$ value $=1.00$ (not significant) } \\
\hline \multicolumn{5}{|c|}{ Subvalvular change } \\
\hline 1 & 11 & $(18.3)$ & 14 & $(23.3)$ \\
\hline 2 & 26 & $(43.3)$ & 29 & $(48.3)$ \\
\hline 3 & 22 & $(36.7)$ & 17 & $(28.3)$ \\
\hline 4 & 1 & $(1.7)$ & 0 & \\
\hline Mean \pm SD & \multicolumn{2}{|c|}{$2.22+0.76$} & \multicolumn{2}{|c|}{$2.05+0.72$} \\
\hline \multicolumn{5}{|c|}{$P$ value $=0.221$ (not significant) } \\
\hline \multicolumn{5}{|c|}{ Calcification } \\
\hline 0 & 39 & $(65.0)$ & 33 & $(55.0)$ \\
\hline 1 & 13 & $(21.7)$ & 22 & $(36.7)$ \\
\hline 2 & 6 & $(10.0)$ & 5 & $(8.3)$ \\
\hline 3 & 2 & $(3.3)$ & 0 & \\
\hline $\mathrm{P}$ value $=0.901$ (not significant $)$ & \multicolumn{2}{|c|}{$0.52 \pm 0.81$} & \multicolumn{2}{|c|}{$0.53 \pm 0.65$} \\
\hline $\begin{array}{l}\text { Mean_SD } \\
P \text { value }=0\end{array}$ & \multicolumn{2}{|c|}{$6.43 \pm 1.53$} & \multicolumn{2}{|c|}{$6.30 \pm 1.33$} \\
\hline
\end{tabular}


Table-V

Relationship between total Wilkins' score and total commissural morphology score among the patients

\begin{tabular}{lccc}
\hline \multirow{2}{*}{$\begin{array}{l}\text { Total } \\
\text { commissural } \\
\text { morphology }\end{array}$} & $\begin{array}{c}\text { PTMC (n=60) } \\
\text { (Mean } \pm \text { SD) }\end{array}$ & $\begin{array}{c}\text { CMC (n=60) } \\
\text { (Mean } \pm \text { SD) }\end{array}$ & P value \\
\hline $\begin{array}{l}\text { Good commissural } \\
\text { morphology (0-1) }\end{array}$ & $5.23 \pm 1.19$ & $5.67 \pm 1.12$ & $0.163 \mathrm{NS}$ \\
$\begin{array}{l}\text { Bad commissural } \\
\text { morphology (2-3) }\end{array}$ & $7.13 \pm 1.26$ & $7.25+1.03$ & $0.70 \mathrm{ONS}$ \\
\hline
\end{tabular}

NSNot significant

Distribution of two different echo-scores are statistically not significant between two groups but wellcorrelated between two echo-scoring systems $(r=0.71)$

Table-VI

Distribution of echocardiographic score of commissural morphology of mitral valve among the patients

\begin{tabular}{|c|c|c|c|c|}
\hline \multirow{2}{*}{$\begin{array}{l}\text { Commissural } \\
\text { morphology }\end{array}$} & \multicolumn{2}{|c|}{ PTMC $(n=60)$} & \multicolumn{2}{|c|}{ CMC $(\mathrm{n}=60)$} \\
\hline & Frequency & $(\%)$ & Frequency & $(\%)$ \\
\hline \multicolumn{5}{|c|}{$\overline{\text { Commissural fusion }(\mathrm{F})}$} \\
\hline 0 & 8 & (13.3) & 9 & $(15.0)$ \\
\hline 1 & 52 & $(86.7)$ & 51 & $(85.0)$ \\
\hline Mean \pm SD & $0.87 \pm 0.34$ & $0.85 \pm 0.23$ & & \\
\hline \multicolumn{5}{|c|}{$\mathrm{P}$ value $=0.793$ (not significant) } \\
\hline \multicolumn{5}{|c|}{ Commissural thickness $(\mathrm{T})$} \\
\hline 0 & 21 & $(35.0)$ & 35 & $(58.3)$ \\
\hline 1 & 39 & $(65.0)$ & 25 & (41.7) \\
\hline Mean+SD & \multicolumn{2}{|c|}{$0.65 \pm 0.45$} & \multicolumn{2}{|c|}{$0.41 \pm 0.35$} \\
\hline \multicolumn{5}{|c|}{$\mathrm{P}$ value $=0.522$ (not significant) } \\
\hline \multicolumn{5}{|c|}{ Commissural. calcification (C) } \\
\hline 0 & 44 & (73.3) & 47 & (78.3) \\
\hline 1 & 16 & (26.7) & 13 & (21.7) \\
\hline $\begin{array}{l}\text { Mean } \pm S D \\
P \text { value }=0.52\end{array}$ & \multicolumn{2}{|c|}{$0.27 \pm 0.45$} & \multicolumn{2}{|c|}{$0.21 \pm 0.52$} \\
\hline $\begin{array}{l}\text { Mean } \pm \text { SD } \\
{ }^{\prime} \text { v value }=0.1\end{array}$ & \multicolumn{2}{|c|}{$1.78 \pm 0.87$} & \multicolumn{2}{|c|}{$1.48 \pm 0.83$} \\
\hline
\end{tabular}

\section{Table-VII}

Relationship between pre and post PTMC mitral valve area (MVA) and total Wilkins' score, and total commissural morphology

\begin{tabular}{lcc}
\hline Echo score & $\begin{array}{c}\text { Pre PTMC } \\
\text { MVA }\left(\mathrm{cm}^{2}\right)\end{array}$ & $\begin{array}{c}\text { Post PTMC } \\
\text { MVA }\left(\mathrm{cm}^{2}\right)\end{array}$ \\
\hline Total Wilkins' score & & \\
$\quad<6$ & $0.82+0.19$ & $2.02+0.33$ \\
$\quad \geq 6$ & $0.79+0.14$ & $1.91+0.19$ \\
P value & $0.450 \mathrm{NS}$ & $0.050^{*}$ \\
Total commissural morphology (0 3) & & \\
Good (0 1) & $0.90+0.18$ & $2.00+0.31$ \\
Bad (2 3) & $0.84+0.14$ & $1.90+0.19$ \\
P value & $0.119 \mathrm{NS}$ & $0.050^{*}$ \\
\hline
\end{tabular}

*Statistically significant NS Statistically not. significant

Both the total Wilkins' scores (<6 vs $\geq 6$ ) and total commissural morphology scores (good vs bad) were well correlated with postprocedural MVA (r 0.71),. but not with the preprocedural mitral valve area. 


\section{Table-VIII}

Relationship between pre and post CMC mitral valve area (MVA) and total Wilkins' echo score, and total commissural morphology

\begin{tabular}{lcc}
\hline Echo score & $\begin{array}{c}\text { Pre CMC } \\
\text { MVA }\left(\mathrm{cm}^{2}\right)\end{array}$ & $\begin{array}{c}\text { Post CMC } \\
\text { MVA }\left(\mathrm{cm}^{2}\right)\end{array}$ \\
\hline Total Wilkins' score & & \\
$\quad<6$ & $0.80+0.15$ & $1.93 \pm 0.22$ \\
$\quad$ 6 & $0.78+0.15$ & $1.85+0.36$ \\
$\quad$ P value & $0.768^{\mathrm{NS}}$ & $0.050^{*}$ \\
Total commissural morphology (0 3) & & \\
Good (0 1) & $0.85+0.16$ & $2.20+0.24$ \\
Bad (2 3) & $0.79+0.13$ & $1.94+0.24$ \\
P value & $0.965^{\mathrm{NS}}$ & $0.050^{*}$ \\
\hline
\end{tabular}

*Statistically significant ${ }^{\text {NS }}$ Statistically not significant

Both the total Wilkins' scores (<6 vs $\geq 6$ ) and total commissural morphology scores (good vs bad) were well correlated with postprocedural MVA $(r=0.71)$, but not with the preprocedural mitral valve area.

Table-IX

Relationship between preprocedural different echo scores of mitral valve morphology and other selected variables with postprocedural mitral valve area

\begin{tabular}{|c|c|c|c|c|c|c|}
\hline \multirow[b]{3}{*}{$\begin{array}{l}\text { Baseline } \\
\text { variables }\end{array}$} & \multicolumn{6}{|c|}{ Postprocedural mitral valve area $\left(\mathrm{cm}^{2}\right)$} \\
\hline & \multicolumn{3}{|c|}{$<2 \mathrm{~cm}^{2}$} & \multicolumn{3}{|c|}{$>2 \mathrm{~cm}^{2}$} \\
\hline & $\begin{array}{l}\text { PTMC } \\
\text { No.(\%) }\end{array}$ & $\begin{array}{c}\text { CMC } \\
\text { No. }\end{array}$ & $\begin{array}{c}\text { Total } \\
\text { No. }\end{array}$ & $\begin{array}{c}\text { PTMC } \\
\text { No. }\end{array}$ & $\begin{array}{c}\text { CMC } \\
\text { No. }\end{array}$ & $\begin{array}{c}\text { Total } \\
\text { No. }\end{array}$ \\
\hline \multicolumn{7}{|c|}{ Wilkins' total score } \\
\hline$<6$ & $\begin{array}{c}8 \\
(44.4)\end{array}$ & $\begin{array}{c}10 \\
(55.6)\end{array}$ & $\begin{array}{c}18 \\
(25.7)\end{array}$ & $\begin{array}{c}8 \\
(57.1)\end{array}$ & $\begin{array}{c}6 \\
(42.9)\end{array}$ & $\begin{array}{l}14 \\
(28.0)\end{array}$ \\
\hline$\geq 6$ & $\begin{array}{c}26 \\
(50.0)\end{array}$ & $\begin{array}{c}26 \\
(50.0)\end{array}$ & $\begin{array}{c}52 \\
(74.3)\end{array}$ & $\begin{array}{c}18 \\
(50.0)\end{array}$ & $\begin{array}{c}18 \\
(50.0)\end{array}$ & $\begin{array}{l}36 \\
(72.0)\end{array}$ \\
\hline \multicolumn{6}{|c|}{ Total commissural morphology } & $0.650^{\mathrm{NS}}$ \\
\hline Good (0 - 1) & $\begin{array}{c}13 \\
(37.1)\end{array}$ & $\begin{array}{c}22 \\
(62.9)\end{array}$ & $\begin{array}{c}35 \\
(50.0)\end{array}$ & $\begin{array}{c}9 \\
(39.1)\end{array}$ & $\begin{array}{c}14 \\
(60.9)\end{array}$ & $\begin{array}{c}23 \\
(46.0)\end{array}$ \\
\hline $\operatorname{Bad}(2-3)$ & $\begin{array}{c}21 \\
(60.0)\end{array}$ & $\begin{array}{c}14 \\
(40.0)\end{array}$ & $\begin{array}{c}34 \\
(50.0)\end{array}$ & $\begin{array}{c}17 \\
(63.0)\end{array}$ & $\begin{array}{c}10 \\
(37.0)\end{array}$ & $\begin{array}{c}27 \\
(54.0)\end{array}$ \\
\hline $\begin{array}{l}\text { P value } \\
\text { Total MR ec }\end{array}$ & & & $0.155^{\mathrm{NS}}$ & & & $0.098^{\mathrm{NS}}$ \\
\hline Good $<5)$ & $\begin{array}{c}15 \\
(40.5)\end{array}$ & $\begin{array}{c}22 \\
(59.5)\end{array}$ & $\begin{array}{c}37 \\
(52.9)\end{array}$ & $\begin{array}{c}10 \\
(37.0)\end{array}$ & $\begin{array}{c}17 \\
(63.0)\end{array}$ & $\begin{array}{c}27 \\
(54.0)\end{array}$ \\
\hline $\operatorname{Bad}(\geq 5)$ & $\begin{array}{c}19 \\
(57.6)\end{array}$ & $\begin{array}{c}14 \\
(42.4)\end{array}$ & $\begin{array}{c}33 \\
(47.1)\end{array}$ & $\begin{array}{c}16 \\
(69.6)\end{array}$ & $\begin{array}{c}7 \\
(30.4)\end{array}$ & $\begin{array}{c}23 \\
(46.0)\end{array}$ \\
\hline$P$ value & & & $0.676^{\mathrm{NS}}$ & & & $0.624^{\mathrm{NS}}$ \\
\hline
\end{tabular}


Table-X

Relationship between baseline variables with postprocedural reduction of mean pressure gradient (MPG) across the mitral valve

\begin{tabular}{|c|c|c|c|c|c|c|}
\hline \multirow[b]{3}{*}{$\begin{array}{l}\text { Baseline } \\
\text { variables, }\end{array}$} & \multicolumn{6}{|c|}{ Postprocedural mean pressure gradient (mmHg) } \\
\hline & \multicolumn{3}{|c|}{$<6 \mathrm{mmHg}$} & \multicolumn{3}{|c|}{$\geq 6 \mathrm{mmHg}$} \\
\hline & $\begin{array}{l}\text { PTMC } \\
\text { No.(\%) }\end{array}$ & $\begin{array}{c}\text { CMC } \\
\text { No.(\%) }\end{array}$ & $\begin{array}{c}\text { Total } \\
\text { No.(\%) }\end{array}$ & $\begin{array}{l}\text { PTMC } \\
\text { No.(\%) }\end{array}$ & $\begin{array}{c}\text { CMC } \\
\text { No.(\%) }\end{array}$ & $\begin{array}{c}\text { Total } \\
\text { No.(\%) }\end{array}$ \\
\hline \multicolumn{7}{|c|}{ Wilkins' total score } \\
\hline$<6$ & $\begin{array}{c}10 \\
(51.2)\end{array}$ & $\begin{array}{c}11 \\
(48.8)\end{array}$ & $\begin{array}{c}21 \\
(33.9)\end{array}$ & $\begin{array}{c}6 \\
(545)\end{array}$ & $\begin{array}{c}5 \\
(455)\end{array}$ & $\begin{array}{c}11 \\
(19.0)\end{array}$ \\
\hline$\geq 6$ & $\begin{array}{c}.21 \\
(51.2)\end{array}$ & $\begin{array}{c}20 \\
(48.8)\end{array}$ & $\begin{array}{c}41 \\
(66.1)\end{array}$ & $\begin{array}{c}23 \\
(48.9)\end{array}$ & $\begin{array}{c}24 \\
(51.1)\end{array}$ & $\begin{array}{c}47 \\
(81.0)\end{array}$ \\
\hline \multicolumn{7}{|c|}{ Total cornmissural morphology } \\
\hline Good (0 - 1) & $\begin{array}{c}12 \\
(41.4)\end{array}$ & $\begin{array}{c}17 \\
(58.6)\end{array}$ & $\begin{array}{c}29 \\
(46.8)\end{array}$ & $\begin{array}{c}10 \\
(34.5)\end{array}$ & $\begin{array}{c}19 \\
(65.5)\end{array}$ & $\begin{array}{c}29 \\
(50.0)\end{array}$ \\
\hline $\operatorname{Bad}(2-3)$ & $\begin{array}{c}19 \\
(57.6)\end{array}$ & $\begin{array}{c}14 \\
(42.4)\end{array}$ & $\begin{array}{c}33 \\
(53.2)\end{array}$ & $\begin{array}{c}19 \\
(65.5)\end{array}$ & $\begin{array}{c}10 \\
(34.5)\end{array}$ & $\begin{array}{c}29 \\
(50.0)\end{array}$ \\
\hline $\begin{array}{l}\text { P value } \\
\text { Total MR ec }\end{array}$ & & & $0.203 \mathrm{NS}$ & & & $0.118^{\mathrm{NS}}$ \\
\hline Good $<5)$ & $\begin{array}{c}13 \\
(36.1)\end{array}$ & $\begin{array}{c}23 \\
(63.9)\end{array}$ & $\begin{array}{c}36 \\
(58.1)\end{array}$ & $\begin{array}{c}12 \\
(42.9)\end{array}$ & $\begin{array}{c}16 \\
(57.1)\end{array}$ & $\begin{array}{c}28 \\
(48.3)\end{array}$ \\
\hline $\operatorname{Bad}(\geq 5)$ & $\begin{array}{c}18 \\
(69.2)\end{array}$ & $\begin{array}{c}8 \\
(30.8)\end{array}$ & $(41.9)^{26}$ & $\begin{array}{c}17 \\
(56.7)\end{array}$ & $\begin{array}{c}13 \\
(43.3)\end{array}$ & $\begin{array}{c}30 \\
(51.7)\end{array}$ \\
\hline P value & & & $0.110^{\mathrm{NS}}$ & & & $0.293^{\mathrm{NS}}$ \\
\hline
\end{tabular}

${ }^{\text {NsNot significant }}$

\section{Discussion}

Although surgical closed mitral commissurotomy is an effective means for treating patients with mitral stenosis with suitable valve morphology and absent LA thrombus, it has come inherent limitation of its own. Dilating stenotic mitral valve by using balloon catheter introduced through venous route and transseptal puncture is an effective alternative to surgical closed mitral commissurotomy. Although started in early 1980s and being used widely throughout the world, Inoue balloon catheter system is the most popular and widely used method for PTMC. For appropriate selected patients, PTMC may be an cost effective alternative to CMC. Our study was aimed at to see the effectiveness of PTMC for treating patients with mitral stenosis compared to CMC in the context of favourable mitral valve morphology.

Comparison of outcome in the context of echocardiographic mitral valve morphology

Post et al. stated that total Wilkins' (MGH), echocardiographic scores were correlated with postprocedural outcome in both procedures significantly $(\mathrm{P}<0.001) .{ }^{5}$ Subscores for leaflet mobility $(\mathrm{P}<0.001)$ and subvalvular disease $(\mathrm{P}<0.001)$ were highly correlated, but the subscores for valvular thickness $(\mathrm{P}=0.91)$ and calcification $(\mathrm{P}=0.29)$ did not correlate.

Fatkin et al. ${ }^{2}$ mentioned that commissural splitting is the dominant mechanism by which mitral valve area is increased with the Inoue balloon techniques and Tubbs' dilator, and it can be predicted by echocardiographic assessment of commissural morphology. Commissural morphology is a better predictor of outcome than is the total Wilkins' mitral valve echo score.

Padial et $a l .^{3}$ mentioned that the new mitral regurgitation echocardiographic score can predict the development of severe mitral regurgitation after PTMC and can be useful in the selection of patients for this technique.In our findings also showed similar results as the above mentioned papers, but did not 
observe any significant difference between PTMC and CMC.

\section{Short term follow up results}

Our patients had been followed for a short period of $22 \pm 6$ weeks due to some unavoidable limitations. All the patients of our initial series $(n=60)$ were supposed to be followed up for at least 24 weeks in this study. But all the patients could not turn up timely at follow up. Our mean follow up time was $22 \pm 6$ weeks. There was no significant reduction of mitral valve area at follow up $(1.94+0.24$ vs $\left.1.90+0.27 \mathrm{~cm}^{2}\right)$. mean pressure gradient also remained stable during this period (mean $+\mathrm{SD}=$ $5.60+2.09 \mathrm{mmHg}$ ). About 90 percent of the patients of both the groups had remained NYHA class I and II. All the patients at follow up reported significant subjective improvement in their exercise capacity and quality of life. Our findings are well in keeping with other relatively short term follow up reports. ${ }^{6,7}$ Repeat cardiac catheterization was not done to document persistent haemodynamic improvement at follow up in our study.

\section{Baseline variables associated with postprocedural outcome}

Pavlides et al. ${ }^{8}$ analyzed 40 baseline demographic, clinical, echocardiographic and haemodynamic variables of 128 consecutive patients with mitral stenosis who underwent PTMC in order to predict long term event free survival, defined as absence of death, mitral valve replacement or repeat PTMC. They found age $(\mathrm{P}=0.03)$, calcium grade $(\mathrm{P}=0.008)$, echo score $(\mathrm{P}=0.0001)$, preprocedural valve area $(\mathrm{P}=0.0007)$ and some other factors which were statistically significant influences of event free survival.

Iung et al. ${ }^{9}$ developed a logistic model from the first 1088 cases and identified the following predictors of immediate results: age $(\mathrm{P}=0.0004)$, echo score $\left(\mathrm{P}<\mathrm{O}^{*} .0001\right)$, preprocedural MVA $(\mathrm{P}<0.0001)$ and effective balloon dilating area (EBDA) $(\mathrm{P}=0.01)$. Vahaniann in this review article described age, previous surgery, valve anatomy and procedural factors as the predictors of the immediate results. Anatoiny, NYHA class, degree of cardiormegaly and quality of initial results are the predictors of the mid term results. ${ }^{10}$

One of the most common predictors of immediate outcome is echocardiographic score and calcification of mitral valve. In our study, we tried to find out whether any baseline variable or variables has strong association with the postprocedural outcome of PTMC and CMC. Our baseline variables were age $<35$ years and $\geq 35$ years, sex $(\mathrm{M} / \mathrm{F})$, total echo score $(<6$ and $\geq 6$ ), baseline good (score 01 ) vs bad (score 23 ), commissural morphology score and total MR echo score ( $<5$ vs $\geq 5$ score as good vs bad) and preprocedural MVA $\left(\leq 0.07\right.$ and $\left.\geq 0.7 \mathrm{~cm}^{2}\right)$. Our dependable variables were increase in MVA, decrease in MPG, PPG, PASP, decrease in LA size and occurrence/worsening of MR grade. By statistical analysis, we found total MV score to be strongly associated with postprocedural outcome in terms of decrease in MPG< increase in MVA and occurrence/worsening of $\mathrm{MR}(\mathrm{P}<0.001)$ in individual group but there was no significant difference of outcome regarding baseline variables between the two procedures. All these findings are similar as reported in other studies. ${ }^{11,12}$ Otherwise, like many other international studies, we found total echo score to most strongly influence the post PTMC outcome. Other factors, we found to influence different dependent variables of the procedure were age, commissural morphology score and MR score. All these findings are well consistent with other studies.

\section{Conclusion:}

In contrast to surgical closed mitral commissurotorny (CMC), percutaneous transvenous mitral commissurotomy (PTMC) produce excellent and comparable early clinical and haernodynarnic improvement that is sustained through mid-term period of follow-up. However, the good results, simplicity of procedure, short hospital staying, better patient acceptance and elimination of drawbacks of thoracotomy and general anaesthesia indicate that PTMC should be the treatment of choice for patients with tight pliable rheumatic mitral stenosis.

\section{Conflict of Interest - None.}

\section{References}

1. Dean LS. 1994. Percutaneous transvenous mitral commissurotomy: A comparison to the closed and open surgical techniques. Cathet Cardiovas Diagn 2(Suppl): 76-81.

2. Fatkin D, Roy P, Morgan JJ, Feneley MP. 1993. Percutaneous balloon mitral valvotomy with the Inoue single-balloon catheter: Commissural morphology as a determinant of outcome. J Am Coll Cardiol 21: 390-7.

3. Padial LR, Freitas N, Sagie A, Newell JB, Weyman AE, Levine RA, et al. 1996. Echocardiography can predict which patients will develop severe mitral regurgitation 
after percutaneous mitral valvulotomy. $J$ Am Coll Cardiol 27: 1225-51.

4. Manabe H. 1986. Conservative surgery of mitral valve disease [special lecture], pp.15-26. In: Proceedings of the second Bangladesh-Japan joint conference on cardiovascular disease, Dhaka: Japan International Cooperation Agency and Bangladesh Cardiac Society.

5. Post JR, Feldman T, Isner J, Herrmann HC. 1995. Inoue balloon mitral valvotomy in patients with severe valvular and subvalvular deformity. J Am Coll Cardiol 25: 1129-36.

6. Herrmann HC, Kleaveland JP, Hill JA, Cowley MJ, Margolis JR, Nocero MA, et al. 1990. The M-heart percutaneous balloon mitral valvuliplasty registry: initial results and early follow-up. J Am Coll Cardiol 15: 1221-6.

7. Bahl VK, Chandra S, Kothari SS, Talwar KK, Sharma $\mathrm{S}$, Kaul U, et al. 1994. Percutaneous transvenous mitral commissurotomy using Inoue catheter in juvenile rheumatic mitral stenosis. Cathet Cardiovas Diagn 2(Suppl): 82-6.
8. Pavlides GS, Nahhas GT, London J. 1997. Predictors of long term event-free survival after PTMC. Am J Cardilol 79: 1370-4.

9. Iung B, Cormier B, Acar J, Vahanian A, Ducimetiere P, Perte JM. 1996. Functional results 5 years after successful PTMC in a series of 528 patients and analysis of predictive factors. J Am Coll Cardiol 27: 407-11.

10. Vahanian A, Cormier B, Iung B. 1994. Percutaneous transvenous mitral commissurotomy using the Inoue balloon: International experience Cathet Cardiovas Diagn 2(Suppl): 8-15.

11. Palacios IF. 1998. Farewell to surgical mitral commissurotomy for many patients. Circulation 97: 223-6.

12. Hemandez R, Ranuclo C, Alfonso F, Goicolea J, Fernandez-Ortiz A, Escaned J, et al. 1990. Long-term clinical and echocardiographic follow-up after percutaneous mitral valvuloplasty with the Inoue balloon. Circulation 99: 1580-6. 